\title{
EVALUACIÓN ESPACIO-TEMPORAL DEL ESTADO TRÓFICO DE UN LAGO DE GRANDES DIMENSIONES USANDO IMÁGENES MODO9GA
}

\section{SPATIO-TEMPORAL ASSESSMENT OF TROPHIC STATE OF A LARGE LAKE USING MODO9GA IMAGES}

\author{
Ricardo Javier Moncayo Eraso', María de Lourdes Bueno Trindade Galo²
}

Fecha de recepción: 11 de junio de 2016

Fecha de revisión: 26 de julio de 2016

Fecha de aprobación: 31 de octubre de 2016

Referencia: R. J. Moncayo Eraso, M. L. B. Trindade Galo (2017). Evaluación espacio-temporal del estado trófico de un lago de grandes dimensiones usando imágenes MOD09GA. Ciencia e Ingeniería Neogranadina, 27 (1), pp. 27-42, DOI: http://dx.doi.org/10.18359/rcin.1893

\section{RESUMEN}

Aunque la relación empírica entre imágenes satelitales y datos recolectados en campo del disco de Secchi ha sido demostrada por estudios previos en cuerpos de agua continentales, la creación de líneas base para el monitoreo espacio-temporal que permita identificar la estacionalidad del Índice de Estado Trófico calculado con el Disco de Secchi (IET-DS) ha sido poco explorado en el monitoreo de grandes lagos. Para realizar un ejemplo práctico de la dinámica espacio-estacional trófica en el embalse de Porto Primavera (Estado de São Paulo - Brasil) a partir de mayo de 2000 hasta abril de 2015, se utilizaron imágenes generadas por el sensor MODIS-Terra, e igualmente, dos trabajos de campo fueron realizados para obtener valores del Disco de Secchi (DS). Estos valores se usaron para ajustar y validar un modelo de correlación entre los valores de reflectancia de la banda centrada en los $645 \mathrm{~nm}$ y el DS. Los resultados indicaron que el modelo tiene un alto coeficiente de determinación $\left(R^{2}=0,80\right)$ y un moderado error asociado a las estimaciones

1. Ingeniero de Sistemas, Máster en Monitoreo y Control Ambiental, Doctor en Ciencias Cartográficas, Docente Investigador Programa de Ingeniería, Institución Universitaria Centro de Estudios Superiores María Goretti (IU-CESMAG), San Juan de Pasto - Colombia, rjmoncayo@iucesmag.edu.co

2. Ingeniera Forestal, Doctora en Ciencias de la Ingeniería Ambiental, Profesora asistente del programa Pos-Graduaçión en Ciencias Cartográficas y de los programas de pregrado en Ingeniería Cartográfica e Ingeniería Ambiental, Universidade Estadual Paulista (UNESP), Presidente Prudente

- São Paulo - Brasil, mlourdes@fct.unesp.br 
(RMSE = 0,47m). El análisis de la variación estacional del (IET-DS) muestra el siguiente patrón: la transparencia del lago es afectada por las estaciones seca y lluviosa. El valor promedio de las estaciones de muestreo localizadas en el lago lo clasifican como oligotrófico, las regiones próximas a la desembocadura del río Pardo, presentan una clasificación eutrófica durante gran parte del año; en las temporadas de verano y otoño esta condición se extiende a casi toda la región norte del lago.

Palabras clave: embalse, espacio-estacional, reflectancia de superficie, teledetección, transparencia.

\section{ABSTRACT}

Although the empirical relationship between satellite imagery and data collected in the field Secchi disk has been demonstrated by previous studies in inland water bodies, creating the basis line for spatiotemporal monitoring to identify seasonality index trophic state calculated with Secchi disk (TSISD) has been little explored in monitoring large lakes. To make a practical example of the spatioseasonal food web in the Porto Primavera reservoir (State of São Paulo Brazil) from May 2000 to April 2015, images generated by the MODISTerra sensor is used, and, also two field works were performed to obtain values Secchi Disk (SD). These values were used to adjust and validate a model of correlation between reflectance values centered on $645 \mathrm{~nm}$ and DS band. The results indicated that the model has a high coefficient of determination $(R 2=0.80)$ and moderate error associated with the estimates (RMSE $=0,47 \mathrm{~m}$ ). The analysis of seasonal variation (IETDS) shows the following pattern: the transparency of the lake is affected by the dry and rainy seasons. The average value of the sampling stations located on the lake classify it as oligotrophic, next to the mouth of the Rio Pardo region, it has an eutrophic classification for a large part of the year; in the summer and fall seasons this condition extends to almost the entire northern region of the lake.

Keywords: reservoir, spatio-seasonal, surface reflectance, remote sensing, transparency.

\section{INTRODUCCIÓN}

La transparencia del agua es un parámetro ampliamente utilizado para evaluar el nivel de eutrofización de un cuerpo de agua [1-3]. Esta variable generalmente se mide con el disco de Secchi (DS) y está relacionada con la transparencia o la profundidad de penetración de la luz en la columna de agua [4-5]. El valor leído por el DS se ve afectado por las algas flotantes y por el material particulado en suspensión, convirtiendo sus medidas en un indicador de la carga total de partículas o de la concentración orgánica disuelta en el agua [6].

En este sentido, una de las ventajas de utilizar el DS como parámetro de evaluación de aguas, es la facilidad que presenta para su medición y sus muestras pueden ser recolectadas y leídas en el campo, sin la necesidad de ser conservadas y utilizar equipos sofisticados de laboratorio $[3,7]$. Sin embargo, el monitoreo y evaluación puntual 
en embalses de gran dimensión o áreas de difícil acceso por medio de técnicas tradicionales, se tornan costosas y la información obtenida es poco representativa para ser extrapolada para todo el ambiente acuático [8-9].

Un enfoque eficaz para realizar el monitoreo sinóptico de la transparencia del agua y del Índice de Estado Trófico medido con el Disco de Secchi (IET-DS) relacionado con este parámetro ópticamente activo, es la teledetección que permite, inclusive, una planeación adecuada de las campañas de campo. Según [3,10-13], la teledetección ofrece la posibilidad de asociar la reflectancia registrada por el sensor con la transparencia del agua, al ser este valor principalmente determinado por los cambios en las cargas totales de partículas, lo cual es útil para obtener información sobre el estado trófico y el flujo de sedimentos en el cuerpo de agua.

Así, para Wu et al. [14], las resoluciones espectrales con mejores resultados para realizar la derivación del DS en aguas continentales (aguas de tipo II), son las bandas localizadas entre los 459 a 479nm y entre los 545 a 565nm, encontrando que también existe un alto coeficiente de determinación al utilizar el intervalo localizado entre los 620 - 670nm [15]. Según el estudio de Wu et al. [14], los sensores Landsat Thematic Mapper (TM) y Moderate resolution Imaging Spectroradiometer (MODIS) son los más adecuados para mapear la dinámica espacial y estacional del parámetro DS en cuerpos de agua continentales.

De los dos sensores, MODIS es el que presenta las mejores características espectrales (36 bandas), radiométricas (12 bits) y su resolución espacial media $(250,500$ y $1.000 \mathrm{~m})$ hace que sea la opción más adecuada para monitorear la dinámica del DS en un embalse superior a las 160 hectáreas [16]. Otra ventaja del sensor
MODIS es la resolución temporal diaria, la cual permite obtener varias imágenes del lugar de estudio en un tiempo relativamente corto, lo que minimiza el riesgo de perder información por factores climatológicos o por errores propios del sensor [17-18]. Con las series temporales que pueden ser generadas por el sensor MODIS es posible analizar la evolución del cuerpo de agua y evaluar sus componentes de tendencia y estacionalidad de la serie temporal generada [19].

\section{1. ÁREA DE ESTUDIOS}

El lugar de estudio está localizado en la línea divisoria entre los estados de São Paulo, Mato Grosso do Sul y Paraná, entre las coordenadas $53-52^{\circ} \mathrm{W}$ y $22-22^{\circ} 30^{\prime} \mathrm{S}$ [20], como se indica en la Figura 1. Esta región formó parte de una extensa planicie aluvial localizada en el curso alto del río Paraná, siendo necesaria la inundación de más del $50 \%$ de este espacio natural para la construcción de esta mega obra [21-22].

Como elementos de la construcción de este embalse, se pueden citar: su represa, que es la más grande del Brasil con una longitud de $10.186 \mathrm{~m}$ y el espejo de agua, resultado del represamiento de las aguas del río Paraná que formó un embalse con un área de $2.250 \mathrm{~km}^{2}$, un volumen acumulado de 20 billones de $\mathrm{m}^{3} \mathrm{de}$ agua. El lago tiene una longitud de $250 \mathrm{~km}$, y se extiende desde la base del embalse de Jupiá, localizado entre las ciudades de Três Lagoas (Mato Grosso do Sul) y Andradina e Castilho (São Paulo) hasta la ciudad de Primavera, localizada en el sur-este del Estado de São Paulo. A pesar de ser el mayor embalse del Brasil, su generación eléctrica es una de las más bajas, alcanzando solamente $1.814 \mathrm{MW}$, lo que resulta en una relación de $0,8 \mathrm{MW} / \mathrm{km}^{2}$ [21]. 


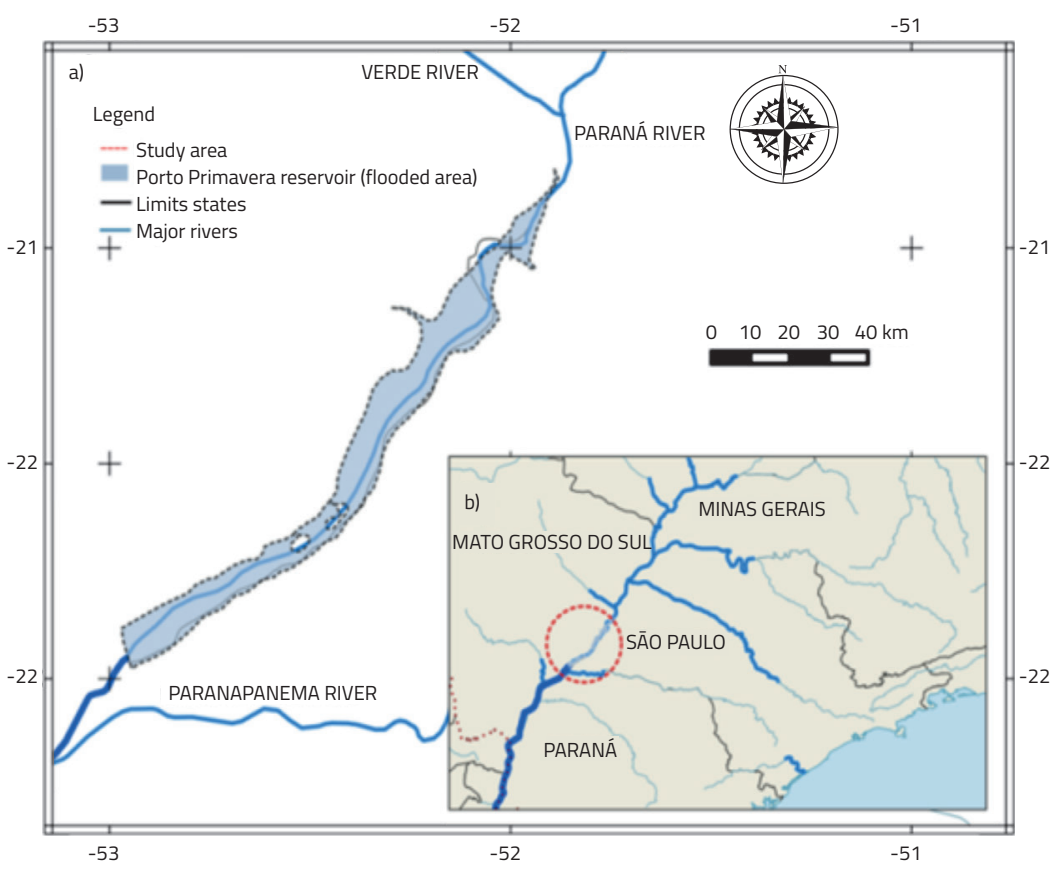

Figura 1. Localización geográfica del embalse de Porto Primavera. (b) indica la cuenca hídrica del embalse. El círculo rojo con líneas discontinuas muestra la localización de Porto Primavera. Las líneas punteadas en color negro señalan el área inundada para la construcción de Porto Primavera.

Fuente: Elaboración propia.

\section{MATERIALES Y MÉTODOS}

\subsection{Desarrollo metodológico}

El estudio integró datos recolectados de la variable limnológica DS y datos generados por el sensor MODIS (MOD09GA) para realizar el mapeo de la dinámica espacial y temporal del DS y del IET-DS; para esto se desarrolló el siguiente procedimiento (véase Figura 2 ):

1. Se generó un repositorio de 1.560 imágenes MODIS (mayo del 2000 a abril de 2015) y se realizó un pre-procesamiento utilizando el software Modis Re-projection Tools (MRT).
2. Se utilizaron las bandas de control de calidad state_ $1 \mathrm{~km}$ y QC_500m para generar imágenes que sólo contengan píxeles clasificados como aguas profundas libres de nubes.

3. Se produjo una imagen de desviación estándar utilizando el índice empírico que emplea los valores de reflectancia leídos por MODIS en la banda $1\left(B_{1}\right)$.

4. Se extrajeron los píxeles que coincidían con la posición geográfica de las estaciones de muestreo y fueron creados los modelos de regresión y validación.

5. Se extrapolaron los modelos encontrados en las imágenes MODIS creando una serie 


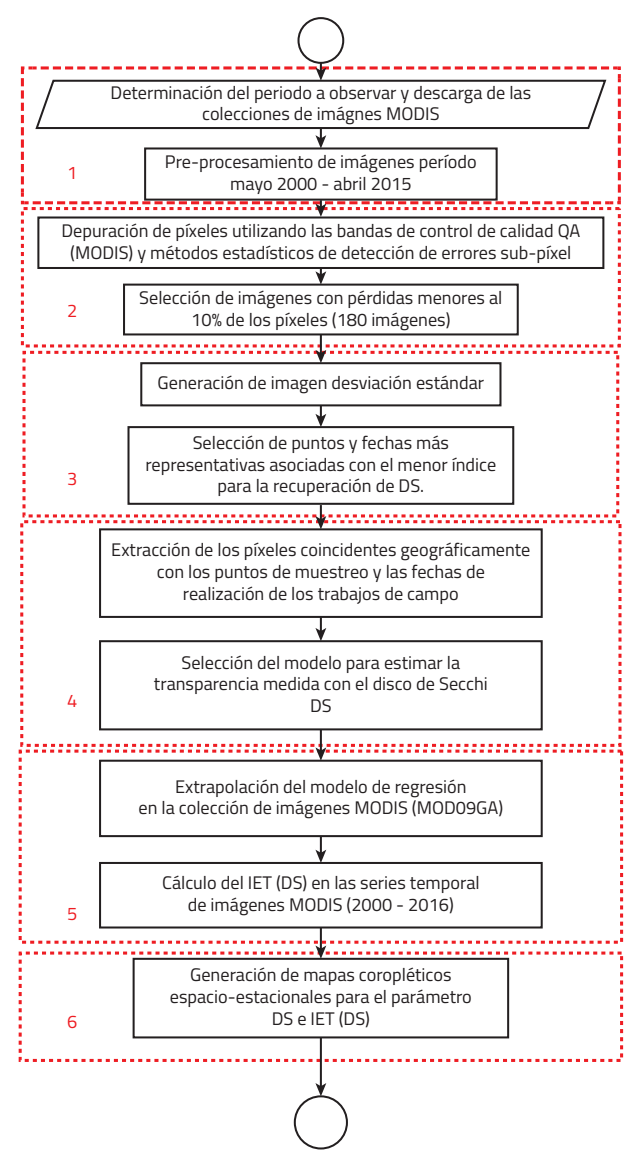

Figura 2. Diagrama de flujo de la metodología implementada, presentando las 6 tareas necesarias para procesar las imágenes MODIS y generar la serie del tiempo estimada de la variable DS e IET (DS).

Fuente: Elaboración propia.

de tiempo con 180 observaciones (15 años: mayo del 2000 a abril de 2015) y con frecuencia de 1 mes.

6. Se generaron los mapas espacio-estacionales de DS y de IET-DS.

\subsection{Datos MODIS (procesamiento de la cole- cción de imágenes MODIS)}

Varios son los productos MODIS de los cuales es posible estimar los valores de la variable óptica- mente activa DS; sin embargo, Knight \& Voth [6] definen que el producto de reflectancia MOD09GA es el más apropiado para recuperar los parámetros relacionados con el proceso trófico y la calidad del agua. MOD09GA generado por la plataforma orbital Terra, es un producto que ya está corregido atmosféricamente de acuerdo con la metodología descrita por Vermote \& Vermeulen [23], y cuenta con resoluciones: espacial de 500m y temporal diaria, que lo hacen adecuado para el monitoreo de cuerpos de agua continentales de grandes dimensiones. 


\subsection{Delineamiento experimental}

Una vez definido el producto MODIS a utilizar, se procedió a realizar la descarga de las imágenes desde el web-site de la United States Geological Survey (USGS)-glovis (http://glovis. usgs.gov/). Un total de 1.065 imágenes fueron seleccionadas para crear una colección de datos temporales que tiene como referencia el intervalo de tiempo: mayo del 2000 a abril de 2015. Las imágenes se procesaron utilizando rutinas desarrolladas en los lenguajes Python y $R$, aplicaciones que dinamizan los procesos de reproyección y recorte espacial y espectral. También permitieron aislar el cuerpo de agua al tener como referencia una máscara en el formato Geotiff previamente creada. Otro proceso realizado con las rutinas fue el de convertir los ND de la imagen original para valores de reflectancia utilizando la ecuación descrita por Rudorff et al. [24] para recuperar valores de reflectancia ( $R$ ) (véase Ecuación 1). Para depurar la información de la banda 1, se utilizaron las bandas de control de calidad QA (State_1km_1, QC_500m_1); con este procedimiento se eliminaron los píxeles con errores en su lectura o contaminados por nubes, y se suprimieron las imágenes con más del 10\% de error del área total del cuerpo de agua estudiado. Como resultado de este proceso, se obtuvo una colección de datos compuesta por 180 imágenes, donde cada una hace referencia a un mes del año, iniciando la serie temporal en mayo del 2000 y terminando en abril de 2015.

$$
P_{i} / 10000
$$

Una vez filtrada la información de la serie temporal, se encontró que existían algunos meses en los cuales no es posible tener una imagen MODIS con menos del $10 \%$ de errores en sus píxeles; para evitar valores nulos en estas posiciones, se usó un método de interpolación de promedio móvil que permite obtener una nueva imagen para cada posición con datos erróneos.

\subsection{Selección de las estaciones de muestreo y fechas para la recolección de datos}

Para definir la posición de las estaciones de muestreo en el embalse de Porto Primavera, así como para encontrar el mejor periodo para realizar las campañas de campo, se utilizó un conjunto de imágenes correspondientes al intervalo mayo del 2000 a abril de 2013, las cuales fueron previamente procesadas de acuerdo la Sección 2.3. Para especificar la localización de los puntos de muestreo, fue considerada la dispersión espacial de la desviación estándar media en un periodo de 13 años, calculada con el índice $\mathrm{B}_{1}$, al considerar la buena correlación que presenta este índice con los valores de DS (véase Figura 3).

\section{5 Índice de estado trófico calculado con el Disco de Secchi (IET-DS)}

El IET permite clasificar los cuerpos de agua en diferentes niveles tróficos, usando una escala de 1 a 100 al derivar los valores de las variables clorofila- $a$, fósforo total y DS. Si un cuerpo de agua no presenta ningún estrés antropogénico, el índice reportará un valor bajo, lo que significa que el ecosistema tiene pocos nutrientes, sedimentos o contaminantes de diferente tipo [25-27]. El índice de estado trófico adoptado para lagos tropicales, es una modificación del índice de Carlson [28] realizada por Toledo et al. [29], que alteró las expresiones originales para adecuarlas a los ambientes tropicales [30]. La ecuación resultante para el IET-DS después de 


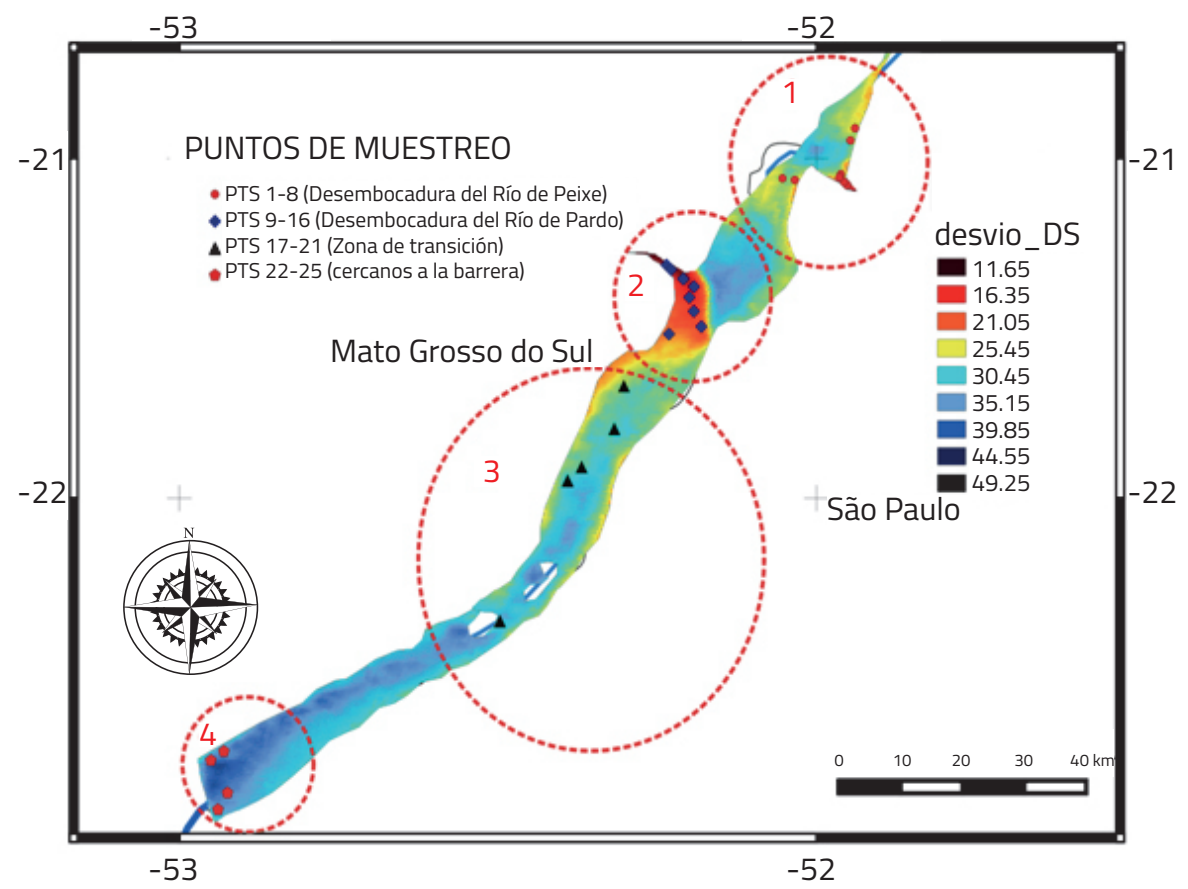

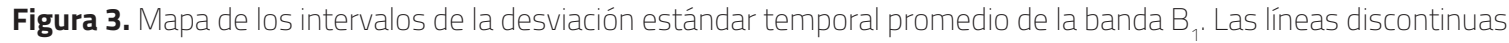
muestran los grupos de puntos de muestreo en el embalse de Porto Primavera.

Fuente: Elaboración propia.

las modificaciones hechas por Toledo, es presentada en (2).

$$
\mathrm{IET}_{(\mathrm{DS})}=10\{6-[\ln (\mathrm{DS}) / \ln (2)]\}
$$

En la Ecuación 2 [30] se tiene:

$D S$ : transparencia medida con el disco de Secchi y expresada en $m$.

In: logaritmo natural.

Para obtener la distribución especial del IET se utilizó la Ecuación (1), la cual considera solamente la medida con el disco de Secchi y su correlación con la banda $1 \mathrm{R}_{(645)}$ del sensor MODIS. El IET-DS se calculó utilizando el índice $\mathrm{R}_{(645)}$ y la Ecuación (2), para extrapolar el IET-DS estimado en cada píxe/ de las imágenes MODIS se utilizó el procedimiento definido por Olmanson et al. [31].

\subsection{Calibración y validación del modelo}

Con el fin de encontrar el mejor modelo de estimación para recuperar la variable DS, se seleccionaron las imágenes con menores errores y con mínima contaminación de nubes en sus píxeles que coincidieran con las fechas de los trabajos de campo; y para tener una mayor 
colección de datos fueron evaluadas las imágenes de la semana posterior y anterior a las campañas de muestreo.

Una vez seleccionadas las mejores imágenes (7 de octubre de 2014 y 17 de abril de 2015) se extrajeron los píxeles de la imagen que coincidían con las coordenadas geográficas de los puntos de muestreo, siguiendo el procedimiento definido por Jönsson \& Eklundh [32]. Posteriormente, los datos de las imágenes fueron divididos en dos grupos, donde los datos y la imagen del mes de octubre sirvieron para calibrar el modelo, los datos y la imagen del mes de abril fueron empleados para validar el modelo encontrado.

Del mismo modo, los modelos encontrados fueron validados utilizando el coeficiente de determinación, el Root Mean-Square Error (RMSE), y siguiendo el procedimiento descrito por Dall'olmo et al. [33], se utiliza la Ecuación (3) para eliminar los valores extremos en las estaciones observadas, los puntos que presentaron un $\varepsilon_{i}>2 \circ \varepsilon_{i}<2$ desviaciones estándar, fueron retirados del conjunto de datos al considerarlos como valores atípicos en las observaciones [33].

$$
\varepsilon_{\mathrm{i}}=100\left(\left(\mathrm{DS}_{\text {prev,i }}-\mathrm{DS}_{\text {med,i }_{\mathrm{i}}}\right) / \mathrm{DS}_{\text {med,i }}\right)
$$

En la Ecuación 3 se tiene:

$\varepsilon_{i}$ : error de la observación $i$

DS $_{\text {prev,i }}$ : DS estimada

$\mathrm{DS}_{\text {med,i }}$ : DS recolectada en campo campo, se siguió el procedimiento descrito por Demir et al. [34], donde los modelos de calibración de la variable DS obtenidos para las imágenes de referencia (octubre de 2014 y abril del 2015) (dominio de origen), fueron extrapolados para las otras 178 imágenes (dominio destino) que conforman la serie de tiempo. Para dinamizar el proceso de extrapolación del modelo de calibración en todas las imágenes de la serie de tiempo, se desarrollaron rutinas en el lenguaje de programación Python.

\section{RESULTADOS}

\subsection{Resultados de los modelos de regresión}

Los valores del conjunto de datos recolectados para la variable DS en octubre de 2014, estuvieron entre los 5,5m y 0,5m; los menores valores fueron leídos en el río Pardo y las máximas lecturas fueron encontradas en los puntos más próximos a la barrera del embalse. El modelo de calibración (véase Figura 4) se genera utilizando las lecturas medidas en campo de DS y la reflectancia leída en $\mathrm{B}_{1}$ del sensor MODIS. Se encontró que para estos dos parámetros pueden ser estimados utilizando un modelo de regresión cuadrático con coeficientes altos en sus estimadores estadísticos $\mathrm{R}^{2}=0,80 ; \mathrm{RMSE}=0,47 ; \mathrm{RRMSE}=0,13$ con $n=21$. La Ecuación 4 que define el modelo de calibración:

$$
\begin{gathered}
D S(m)=-459,4 *\left(R_{1}(645)\right)^{2}+9,005 * \\
\left(R_{1}(645)\right)+2,45
\end{gathered}
$$

\section{En (4) se tiene que:}

$\mathrm{R}_{1}$ : reflectancia leída por el sensor MODIS en la banda centrada en los $645 \mathrm{~nm}$

\subsection{Generación de series temporales medias y puntuales}

Para obtener observaciones históricas ajustadas con los valores leídos en los trabajos de 


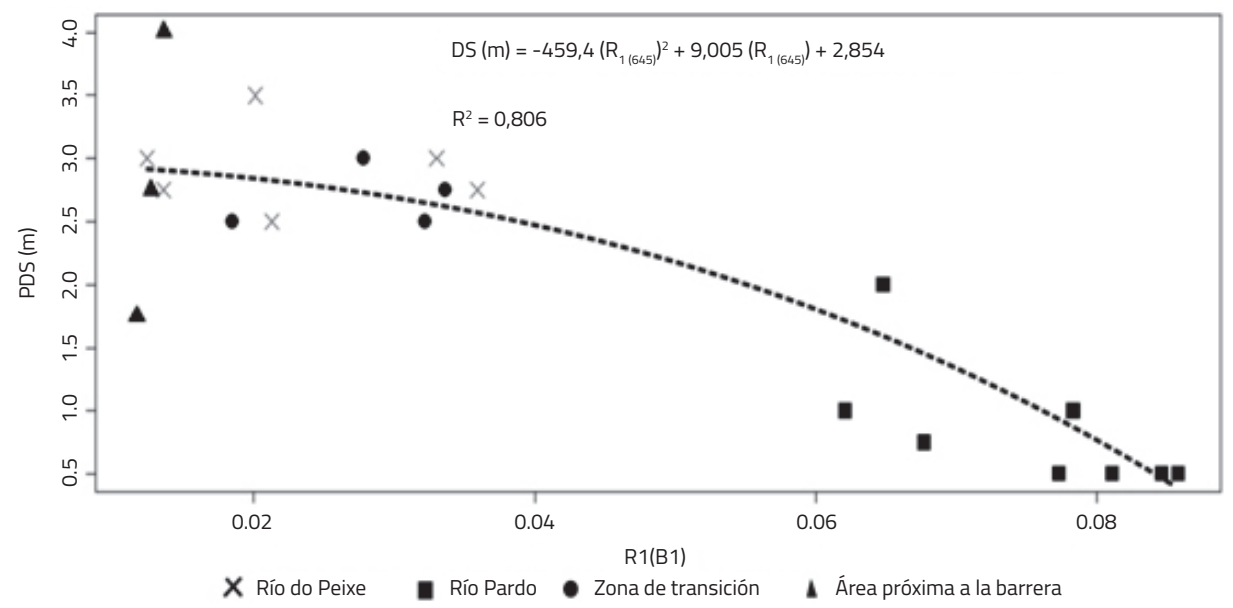

Figura 4. Modelo de calibración para la variable DS. El coeficiente de determinación R² consideró la reflectancia para la banda 1 del producto MODO9 y la medida leída en campo de la variable DS en el mes de octubre de 2014 en 21 elementos de muestreo.

Fuente: Elaboración propia.

El análisis de correlación (modelo de validación) entre los valores medidos en campo en abril y la $R_{1}(645 \mathrm{~nm})$, se hizo con una imagen que no coincidía con los días en los que se realizó el trabajo de campo; esto debido a que las imágenes de los días 13 a 16, tenían píxeles contaminados por nubes o presentaban errores asociados con el funcionamiento del sensor MODIS. Sin embargo, los ND extraídos de la banda 1 del 17 de abril, indicaron que existía un buen coeficiente de determinación después de la eliminación de los puntos que presentaban valores atípicos utilizando el procedimiento definido por Dall'olmo et al. [33].

El modelo de validación (Figura 5) para la recuperación de la variable DS, indicado en la Ecuación 4, definió un coeficiente de determinación entre los valores medidos y estimados igual a $\mathrm{R}^{2}$, 0.46; RMSE, 0,73 m; \%RMSE, 29,21\%. Según Knigth \& Voth [6], estos valores del mode- lo de validación pueden ser considerados como moderados al tener en cuenta que el coeficiente de determinación es mayor al 0,43, sin embargo el RMSE $(0.73 \mathrm{~m})$ asociado al modelo es mucho menor que el relacionado en estos trabajos, para todos los casos indicados por Knigth \& Voth [6] el RMSE es mayor a $1 \mathrm{~m}$.

\subsection{Precisión en la clasificación del IET (DS) para el embalse de Porto Primavera}

Se hizo una validación sobre la precisión del IET (DS) entre el IET calculado con los muestreos de campo y el estimado para cada uno de los píxeles MODIS coincidentes con la posición geográfica de cada punto. Los resultados obtenidos en las regresiones y los descriptores de evaluación así como algunos datos de estadística descriptiva se presentan en la Tabla 1. 


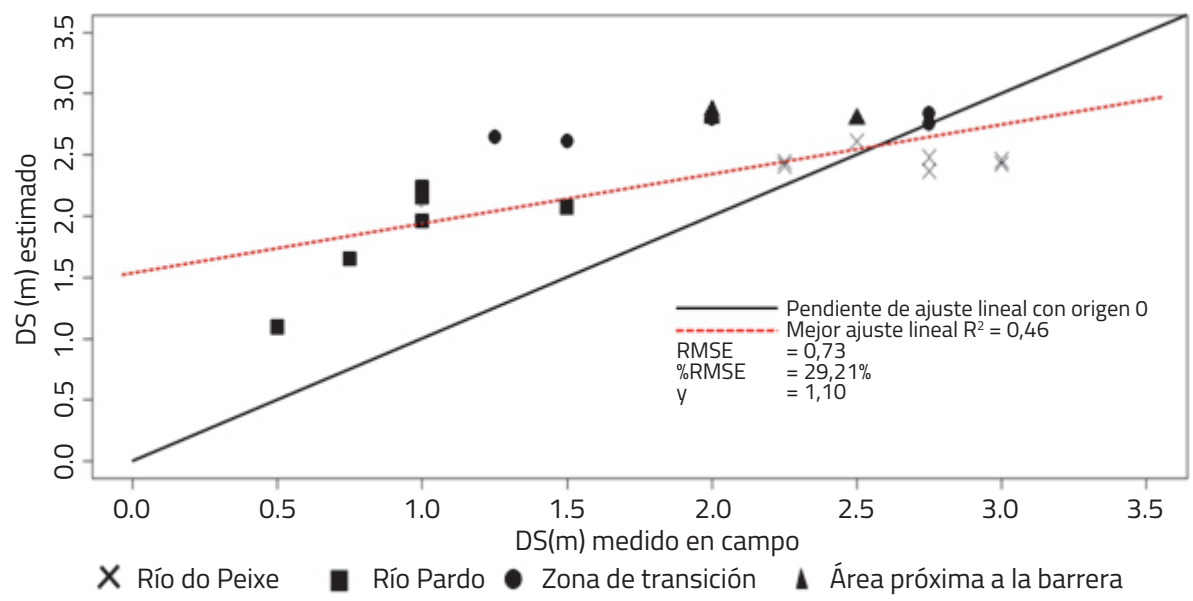

Figura 5. Validación del modelo de calibración para la recuperación de la variable DS ("ecuación (4)"), realizada a partir de los datos de abril de 2015.

Fuente: Elaboración propia.

Tabla 1. Estadística descriptiva de IET calculado y estimado para los campos e imágenes de octubre de 2014 y abril de 2015 .

\begin{tabular}{|c|c|c|c|c|c|}
\hline DS & Media & $\begin{array}{c}\text { Desv. } \\
\text { est }\end{array}$ & Máximo & Mínimo & R \\
\hline Campo & 52,04 & 8,84 & 70 & 40 & \multirow{2}{*}{0,81} \\
\hline Imagen & 49,89 & 8,38 & 80,30 & 44,65 & \\
\hline
\end{tabular}

Fuente: Elaboración propia

Los resultados de la Tabla 1 indican que el coeficiente de correlación entre los valores estimados por la reflectancia de la banda 1 (620 - 670nm) y las mediciones de DS realizadas en campo convertidos ambos valores a IET (DS) corresponden a coeficientes satisfactorios y explican más del $75 \%$ de la variabilidad en el modelo predictivo. Adicionalmente, fue realizado un análisis de la clasificación de los píxeles en la escala del IET (DS), donde se observó que el $45,45 \%$ de los elementos estimados a partir de las imágenes MODIS fueron clasificados de forma correcta. Las diferencias más grandes en la clasificación se presentan en la región del río Pardo. También, se encontraron clasificaciones erróneas en otras áreas que presentaban menor variabilidad (zona norte, transición y áreas próximas a la muralla de la represa). La incertidumbre en las áreas homogéneas se debe al error asociado a los modelos de calibración y validación $(0,47 m ; 0,73 m)$, siendo estos errores suficiente para generar una clasificación errónea entre las categorías oligotrófica y ultraoligotrófica. Los errores presentados en la clasificación de los píxeles, pueden ser ocasionados por no contar con una imagen coincidente con los trabajos de campo realizados en el mes de abril o por errores en las mediciones efectuadas en el lugar de muestreo.

La distribución espacial del IET (DS) para las fechas en las que se llevaron a cabo las cam- 
pañas de muestreo, son presentadas en las Figuras 6a y $6 b$.

La distribución espacial del IET-DS, el día 7 de octubre de 2014, es presentada en la Figura 6a. Para este trabajo en particular, se tiene que aproximadamente el $70 \%$ de los píxeles de la imagen MODIS fue asociado a las clasificaciones ultra-oligotrófica y oligotrófica, $18 \%$ de los píxeles presentó una clasificación mesotrófica y $9,2 \%$ de los píxeles pueden ser categorizados como eutróficos y súper-eutróficos y $0,8 \%$ son píxeles sin clasificación. En general, se observa que el lago presenta condiciones ultra-oligotróficas y oligotróficas en la margen situada en el estado de São Paulo, esto puede ser consecuencia de que la margen paulista es formada por una zona escarpada sin zonas de deposición marginal, al contrario de la extensa zona de planicie aluvial del valle del río Paraná del lado de Mato Grosso do Sul, que favorece la deposición de sedimentos como lo describe Dias [21].

Los valores clasificados como mesotróficos se localizaron principalmente en las desembocaduras del río do Peixe (1), río Taquaraçu (2), Arroyo do Veado (3), río Pardo (4), y Ribeirão Quiteriozinho y río Quiterio (5). En el mapa del 17 de abril de 2015 (véase Figura 6b) se aprecia un incremento en la productividad trófica en las regiones próximas a la desembocadura del río do Peixe (1), clasificando esta área del lago como mesotrófica y en la zona de mayor influencia del río do Peixe el lago puede llegar a tener un grado de eutrófico. Para esta fecha a)

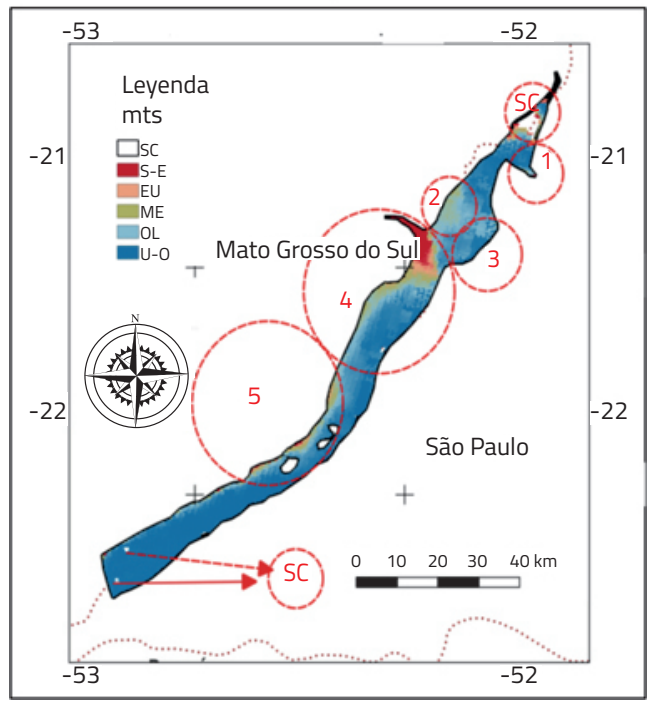

b)

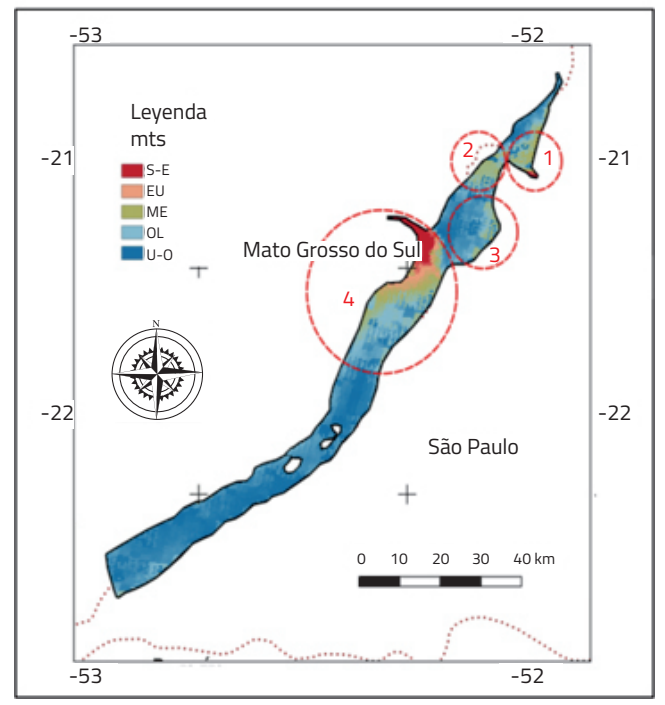

Figura 6. Distribución espacial del IET (DS) estimado utilizando la ecuación 4 sobre dos imágenes MOD09GA, generadas en los días (a) 7 de octubre de 2014 y (b) 17 abril de 2015. En la leyenda se indica las clasificaciones tróficas Súper-eutrófico (S-E), Eutrófico (EU), Mesotrófico (ME), Oligotrófico (OL), Ultra-oligotrófico (U-O).

Fuente: Elaboración propia. 
la productividad trófica también se incrementa en el Arroyo Do Paredão (2), río Pardo (4), Arroyo do Veado (3).

\subsection{Distribución espacial y estacional del IET(DS) en el embalse de Porto Primavera}

La distribución espacial de la variable DS (m), es representada para cada estación del año (primavera, verano, otoño e invierno) en la Figura 7.

El análisis de la variación estacional de la distribución espacial de la variable IET (DS) (véase Figura 7) indica que el índice tiene una alta correlación con las temporadas de lluvia y seca. La temporada de invierno (véase Figura 7d) se caracteriza por bajos valores de IET (DS) (valor medio cercano a 42), excepto en la región de la desembocadura del río Pardo, en la cual los valores promedio clasifican el lago entre mesotrófico y eutrófico durante todo el año.
En la estación de primavera (véase Figura 7a) el IET (DS) aumenta como consecuencia del incremento de las lluvias y de material particulado que es llevado al lago a través de los ríos formadores del lago, mostrando una varianza más significativa en la región próxima al río Pardo y con menor intensidad en las regiones próximas a los ríos do Peixe, Verde y Paraná.

Por su parte, el verano es una estación diferenciada por sus altos valores de IET (DS), principalmente en la zona comprendida entre las desembocaduras de los ríos do Peixe, Pardo y Taquaraçu. En el otoño (véase Figura 7c), el IET (DS) presenta un valor medio para el embalse de 50 .

\section{CONCLUSIONES}

Los productos MOD09GA demuestran tener una alta correlación con las mediciones de DS leídas en campo; en este estudio se encontró

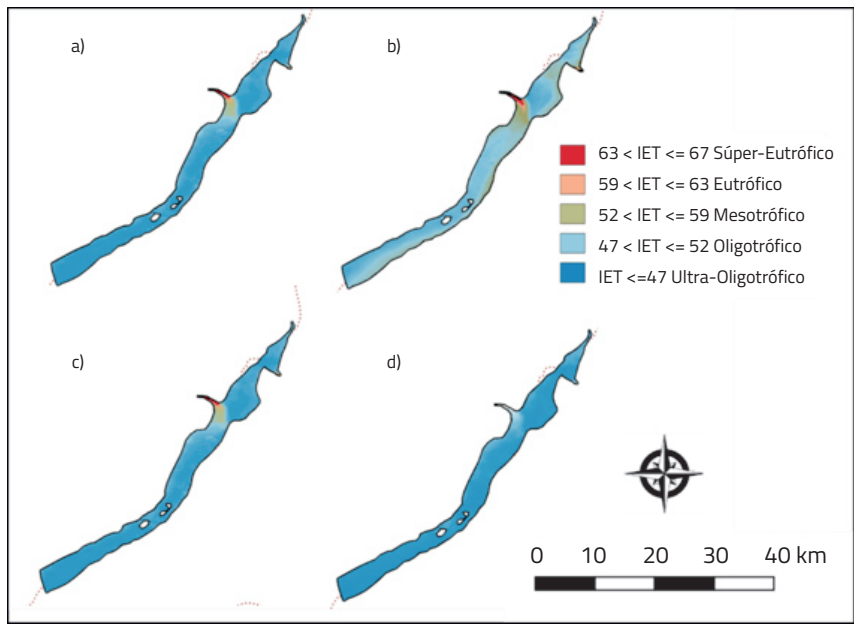

Figura 7. Mapas coropléticos de la distribución espacial de la variable IET (DS) (generadas a partir de la ecuación (4)) en el embalse de Porto Primavera, en cada estación del año: (a) primavera; (b) verano; (c) otoño; (d) invierno.

Fuente: Elaboración propia. 
que se tiene un coeficiente de determinación por encima del $80 \%$ entre los valores leídos con el disco de Secchi y los píxeles extraídos de la imagen MODIS. Igualmente, más del $45 \%$ de los píxeles convertidos al IET (DS) fueron clasificados de forma correcta. Sin embargo, se tiene que para algunas zonas del embalse, el IET (DS) es sobrestimado hasta en 5 unidades para el campo de octubre de 2014 y subestimado en 4 unidades para el campo de abril de 2015. Adicionalmente, para la imagen coincidente con el mes de abril se observa que los valores extraídos de los píxeles coincidentes con las estaciones de muestreo subestiman su valor de IET (DS) en 4.09 unidades (promedio campo 51,63; promedio imagen 47.53); la clasificación errónea ocurrió principalmente en la región de la desembocadura del río Pardo; aun así, los resultados obtenidos son óptimos para poder observar la distribución espacial y temporal del IET (DS) dentro del lago de Porto Primavera.

Estacionalmente, se observa que los ciclos del IET (DS) tienen correspondencia con las temporadas seca y lluviosa; así mismo al encontrar que la temporada de otoño es una estación del año donde se tiene una mayor dispersión espacial del IET (DS).

Finalmente, la alta resolución temporal y media resolución espacial del producto MOD09GA fueron adecuadas para analizar la distribución espacial de la variable DS y realizar una planeación de campo apropiada, donde se dio prioridad a los lugares con mayor probabilidad de tener una lectura baja de DS.

\section{AGRADECIMIENTOS}

Los autores agradecen al programa de PósGraduação en Ciências Cartográficas y al de- partamento de Cartografía de la UNESP - Universidade Estadual Paulista Júlio Mesquita Filho, por haber patrocinado con recursos financieros, infraestructura e instrumentos que permitieron el desarrollo de este proyecto. Al proyecto MODIS por proporcionar el producto MODO9GA; a la FEPAP por el apoyo financiero en los levantamientos de campo. El primer autor agradece a la UNESP y a la AIUP por la beca de Doctorado, y a la IUCESMAG por brindar los medios necesarios para la correcta presentación de este documento.

\section{REFERENCIAS}

[1] McCullough, I., Loftin, C. \& Sader, S. (2012). High-frequency remote monitoring of large lakes with MODIS 500 m imagery. Journal of Remote sensing of environment, 124, pp. 234-241. doi: 10.1016/j. rse.2012.05.018

[2] Butt, M. \& Nazeer, M. (2015). Landsat ETM+ Secchi Disc Transparency (SDT) retrievals for Rawal Lake, Pakistan. Journal Advances in Space Reseach, 56(7), pp. 1428-1440. doi: 10.1016/j. asr.2015.06.041

[3] Gholizadeh, M., Melesse, A. \& Reddi, L. (2016). A comprehensive Review on Water Quality Parameters Estimation Using Remote Sensing Techniques. Journal Sensors, 16(8), pp. 1298-1306. doi: 10.3390/s16081298

[4] Preisendorfer, R. (1986). Secchi disk science: Visual optics of natural waters. Limnology and oceanography, 31, pp. 909926. doi: 10.4319/lo.1986.31.5.0909

[5] Lee, Z., Shang, S., Hu, Ch., Du, K., Weidemann, A., Hou, W., Lin J. \& Lin, G. Secchi disk depth: new theory and 
mechanistic model for underwater visibility. Journal Remote Sensing of Environment, 169, pp. 139-149. doi: 10.1016/j.rse.2015.08.002

[6] Knight, J. \& Voth, M. (2012). Application of MODIS imagery for intra-annual water clarity assessment of Minnesota Lakes. Journal of remote sensing of environment, 4(7), pp. 2181-2198. doi: 10.3390/ rs4072181

[7] Domínguez, J., Chuvieco, E. \& Sastre, A. (2009). Monitoring transparency in inland water bodies using multispectral images. International Journal of Remote Sensing, 30(6), pp. 1567-1586. doi: 10.1080/01431160802513811

[8] Papoutsa, C. \& Hadjimitsis, D. (2013). Remote sensing for water qality surveillance in Inland Waters: The case study of Asprokremmos dam in cyprus. In: D.Hadjimitsis (Ed.), Remote sensing of environment - integrated approaches, pp. 131-153. doi: 10.5772/39308

[9] Lillesand, T., Johnson, W., Deuell, R., Lindstrom, O. \& Meisner, D. (1983). Use of landsat data to predict the trophic state of Minnesota lakes. Photogrammetric engineering and remote sensing, 49(2), pp. 219-229.

[10] Chen, Z.; Hu, C. \& Muller-Karger, F. (2007). Monitoring turbity in tampa bay using MODIS/Aqua 250-m imagery. Journal Remote sensing of environment, 109(2), pp. 207-220. doi: 10.1016/j. rse.2006.12.019

[11] Liu, Y., Islam, A. \& Gao, M. (2003). Quantification of shallow water quallity parameters by means of remote sensing. Progress in physical geography, 27(1), pp. 24-43. doi: 10.1191/0309133303pp357ra

[12] Pozdnyakov, D. \& Grass, H. (2003). Colour of inland and Coastal Waters: Methodology for its interpretation. Chichester: Praxis Publishing. pp.1-330.

[13] Gitelson, A., Gurlin, D., Moses, W. \& Barrow, T. (2009). A bio-optical algorithm for the remote estimation of the chlorophyll-a concentration in case 2 waters. Environmental Research Letters, 4, pp. 1-5. doi: 10.1088/17489326/4/4/045003

[14] Wu, G., De Leeuw, J., Skidmore, A., Prins, H. \& Liu, Y. (2008). Comparison of MODIS and landsat TM5 images for mapping tempo-spatial dynamics of Secchi disk depths in Poyang Lake National Nature Reserve, China. International Journal of Remote Sensing, 29(8), pp. 2183-2198. doi: 10.1080/01431160701422254

[15] Dlamini, S., Nhapi, I., Dumindoga, W., Nhiwatiwa, T. \& Dube, T. (2016). Assessing the feasibility of integrating remote sensing and in-situ measurements in monitoring water quality status of Lake Chivero, Zimbawe. Journal Physics and Chemistry of the Earth, Parts $A / B / C, 93, \mathrm{pp}$. 2-11. doi: 10.1016/j.pce.2016.04.004

[16] Novo, E., Barbosa, C.; Melack, J., De Freitas R., Titonelli, F. \& Shimabukuro, Y. (2007). Comparação de Imagens MODIS e ETM+ para Estudo de Águas Interiores: Imposições da Resolução Espacial. Revista Brasileira de Cartografia, 58(2), pp. 109-118.

[17] Koponen, S., Pulliainen, J., Kallio, K., Vepsäläinen, J. \& Hallikainen, M. (2001). Use of MODIS data for monitoring turbidity in Finnish Lakes. In IEEE 2001 International Geoscience and Remote Sensing Symposium. doi: 10.1109/ IGARSS.2001.977943

[18] Kim, S., Y, Ch. \& Ouchi. (2015). Spatiotemporal patterns of Secchi depth in the waters around the Korean Peninsula using MODIS data. Journal Estuarine, 
Coastal and Shelf Science, 164, pp. 172182. doi: 10.1016/j.ecss.2015.07.003

[19] Zhang, Y., Lin, S., Qian, X., Wang, Q., Qian, Y., Liu, J. \& Ge, Y. (2010b). Temporal and spatial variability of chlorophyll a concentration in Lake Taihu using MODIS time-series data. Hydrobiologia, 661(1), pp. 235-240. doi: 10.1007/s10750-0100528-9

[20] Rodríguez, I., Train, S., Bovo-Scomparin, V., Jati, S., Borsalli, C. \& Marengoni, E. (2009). Interannual variability of phytoplankton in the main rivers of the Upper Paraná River floodplain, Brazil: Influence of upstream reservoirs. Brazilian Journal of Biology, 69 (2), pp. 501-516. doi: 10.1590/s151969842009000300006

[21] Dias, J. (2003). A construção da paisagemnaraiadivisória São PauloParaná-MatoGrosso do sul: Um estudo por teledetecção. (Tese Doutorado em Geografia, 274f). Departamento de Geografia, Faculdade de Ciências e Tecnología UNESP, Universidade Estadual Paulista, Presidente Prudente.

[22] Souza Filho, E., Zanetti, K., Pinese, J. \& França, V. (2010). A hidroquímica do rio Paraná após a barrage de Porto Primavera. Boletín de Geografía, 28(2), pp. 101-112. doi: 10.4025/bolgeogr. v28i2.10369

[23] Vermote, E. \& Vermeulen, A. (1999). MODIS algorithm technical background document, atmospheric correction algorithm: Spectral reflectances (MOD09), Version 5.0; recuperado de: http://modis. gsfc.nasa.gov/data/atbd/atbd_mod08. pdf

[24] Rudorff, B., Shimabukuro, Y. \& Ceballos, J. (2007). O sensor MODIS e suas aplicações ambientais no brasil. São José dos Campos, SP: INPE, pp. 55-120.

[25] Kordi, H., Hoseini, S., Sudagar, M. \&
Alimohammadi, A. (2012). Correlation of Chlorophyll-A with Secchi Disk Depth and Water Turbidity in Aquaculture Reservoirs A Case Study on Mohammadabad Reservoirs, Gorgan, Iran. World Journal of Fish and Marine Sciences, 4(4), pp. 340-343. doi: 10.5829/idosi. wjfms.2012.04.04.6345

[26] Barbosa, F., de Freitas, P., Carrhá, R., Paulino, W. \& Tédde, S. (2013). Influence of rainfall on the trophic status of a Brazilian semiarid reservoir. Acta Scientiarum. Biological Sciences, 35(4), pp. 505-511. doi: 10.4025/actascibiolsci. v35i4.18261

[27] Lemley, D., Adams, B., Taljaard, S. \& Strydom, N. (2015).Towards the classification of eutrophic condition in estuaries. Journal Estuarine, Costal and Shelf Science, 164, pp. 221-232. doi: 10.1016/j.ecss.2015.07.033

[28] Carlson, R. (1977). A trophic state index for lakes. Journal of limnology and oceanography, 22, pp. 361-369. doi: 10.4319/lo.1977.22.2.0361

[29] Toledo Jr. A., Talarico, M., Chinez, S. \& Agudo, E. (1983). "Aplicação de modelos simplificados paraa avaliação de processo da eutrofização em lagos e reservatórios tropicais". Presentado en Anais do 12 Congresso Brasileiro de Engenharia Sanitária. Camboriú, Associação Brasileira de Engenharia Sanitaria, Camboriu (SC).

[30] Lamparelli, M. (2004). Graus de trofia em corpos d' água do Estado de São Paulo: avaliação dos métodos de monitoramento. (Tese Doutorado em Ciências Biológicas, 238f). Universidade de São Paulo - USP, São Paulo.

[31] Olmanson, L., Kloiber, S., Bauer, M. \& Brezonik, P. (2001). Image processing protocol for regional assessments of lake water quality. Minnesota, MN: Water 
Resources Center and Remote Sensing Laboratory, pp. 33-82.

[32] Jönsson, P. \& Eklundh, L. (2004). Timesat - a program for analyzing time-series of satellite sensor data. Computers \& Geosciences, 30(8), pp. 833-845. doi: 10.1016/j.cageo.2004.05.006

[33] Dall'olmo, G., Gitelson, A., Rundsquist, D., Leavitt, B., Barrow, T. \& Holz, J. (2005). Assessing the potential of SeaWIFS and MODIS for estimating chlorophyll concentration in turbid productive waters using red near-infrared bands. Journal of Remote sensing of environment, 96, pp. 176-187. doi: 10.1016/j.rse.2005.02.007 [34] Demir, B., Bovolo, F. \& Bruzzone, L. (2013). Classification of time series of multispectral images with limited training data. IEEE Transactions on Image Processing, 22(8), pp. 3219-3233. doi: 10.1109/TIP.2013.2259838 